Pacific Journal of Mathematic 


\section{MEASURES WITH CONTINUOUS IMAGE LAW}

\section{Sun MaN Chang}

Let $M$ be a topological space, and $X$ a metric space. Let $P(X)$ denote the collection of probability measures on $X$. Let $C(M, X)$ denote the set of continuous functions from $M$ to $X$. Let $P(X)$ have the weak topology, and let $C(M, X)$ have the topology of uniform convergence. For a fixed measure $\mu \in P(C(M, X))$, and a member $t \in M$, define a measure $t \mu$ on $X$ by

$$
t \mu(A)=\mu\{f \in C(M, X): f(t) \in A\} .
$$

In this paper, we consider the following problem: given a continuous function $T: M \rightarrow P(X)$, when is there a measure $\mu \in P(C(M, X))$ such that $T(t)=t \mu$ for all $t$ ?

This problem has been introduced and studied by Blumenthal and Corson in [2] and [3].

The main results of this paper are as follows:

1. Let $F$ be a closed subset of a totally disconnected compact metric space $M$, and let $i_{F}: F \rightarrow M$ be the natural inclusion. Let $X$ be a complete separable metric space. Suppose that $T$ is a continuous function from $M$ to $P(X)$, and $\mu_{F}$ is a measure in $P(C(F, X))$ such that $T(t)=t \mu_{F}$ for all $t \in F$. Then there is a measure $\mu \in P(C(M, X))$ such that $T(t)=t \mu$ for all $t \in M$ and $i_{F} \mu=\mu_{F}$. Consequently, the natural map $\phi: P(C(M, X)) \rightarrow C(M, P(X))$, defined as $\phi(\mu)(t)=t \mu(\mu \in P(C(M, X)), t \in M)$ is open, and there is a continuous function $\xi: C(M, P(X)) \rightarrow P(C(M, X))$ such that $\xi(T) \in \phi^{-1}(T)$.

2. Let $M$ be a separable metric space, and let $S^{1}$ be the unit sphere in $R^{2}$. Let $T$ be a continuous function from $M$ into $P\left(S^{1}\right)$ such that Supp $T(t)$ is a connected subarc of $S^{1}$ and of arc length $\leqq 2 \pi-k$, for all $t$ and some fixed $0<k \leqq 2 \pi$. Then there is a measure $\mu \in P\left(C\left(M, S^{1}\right)\right)$ such that $T(t)=t \mu$ for all $t$.

3. Some generalizations of the results in [2] and [3] will be considered.

1. Introduction. Let $Y$ be a metric space. By a measure on $Y$, we mean a regular Borel measure on the class of all Borel subsets of $Y$. Let $\mu$ be a measure on $Y$. We define the support of $\mu$ to be the smallest nonempty closed subset $F \subseteq Y$ such that $\mu(U)>0$ for every open subset $U$ in $Y$ such that $U \cap F \neq \varnothing$. We will denote this 
by $\operatorname{Supp} \mu$. For a fixed $y \in Y$, we denote by $\varepsilon_{y}$ the unit point-mass measure putting mass 1 at the point $y$. Let $C(Y)$ denote the collection of all bounded continuous real-valued functions on $Y$. Let $P(Y)$ denote the collection of all measures on $Y$ with total mass 1 . Let $P(Y)$ have the weak topology as functionals on $C(Y)$. A measure in $P(Y)$ will be called a probability measure.

Let $X$ be a metric space, and let $F \subseteq P(X)$. We call $F$ tight if for every $\varepsilon>0$, there is a compact set $K \subseteq X$ such that $\mu(K) \geqq 1-\varepsilon$ for all $\mu \in F$. If $F=\{\mu\}$, we call $\mu$ a tight measure. Denote by $P_{\tau}(X)$ the collection of all tight measures in $P(X)$ with the relative topology. We call a metric space a Prohorov space if every compact subset of $P_{\tau}(X)$ is tight. Note that every complete metric space is Prohorov [1], and $P_{\tau}(X)=P(X)$ whenever $X$ is complete separable metric.

Now consider a topological space $M$, and a metric space $X$. Let $C(M, X)$ denote the set of continuous functions from $M$ into $X$. Let $C(M, X)$ have the topology of uniform convergence. Note that if $M$ is not compact, the topology on $C(M, X)$ depends on the particular bounded metric used for $X$.

We will use the following notation throughout this paper. Let $Y$ and $X$ be metric and let $\pi$ be a continuous function from $Y$ into $X$. Then $\pi$ induces a mapping, also denoted by $\pi$, from $P(Y)$ to $P(X)$, and defined by $\pi \mu(A)=\mu\left(\pi^{-1}(A)\right)$. Also for $t \in M$, denote simply by $t$ the mapping $f \rightarrow f(t)$ from $C(M, X)$ into $X$. Hence the mapping $\mu \rightarrow t \mu$ from $P(C(M, X))$ to $P(X)$ is defined and continuous by taking $Y$ to be $C(M, X)$ and $t$ to be $\pi$ in the definition of $\mu \rightarrow \pi \mu$ above

Recall that, by a totally disconnected space, we mean a space which has a base consisting of sets which are both open and closed. By a Peano space, we mean a space which is a continuous image of the unit interval. For convenience, we will call a complete separable metric space a Polish space throughout this paper.

In this paper, we consider the following problem: given a continuous function $T: M \rightarrow P(X)$, when is there a measure $\mu \in P(C(M, X))$ such that $T(t)=t \mu$ for all $t$ ? When this happens, we will say that $\mu$ represents $T$.

This problem was introduced by Blumenthal and Corson. They showed that such a measure exists in the following two cases:

(a) $M$ is a compact totally disconnected space, and $X$ is a complete metric space [2].

(b) $M$ is a compact metric space, $X$ is a Peano space, and Supp $T(t)=X$ for all $t$ [3].

In $\S 2$, we let $M$ be a totally disconnected compact metric 
space, the Cantor set for example, and let $X$ be a Polish space. For a fixed $\mu \in P(C(M, X))$, one checks easily that $t \rightarrow t \mu$ is a continuous function from $M$ into $P(X)$. Now define a mapping $\phi: P(C(M, X)) \rightarrow C(M, P(X))$ by

$$
\phi(\mu)(t)=t \mu, \quad \mu \in P(C(M, X)), \quad t \in M .
$$

Blumenthal and Corson (a) showed that $\phi$ maps $P(C(M, X))$ onto $C(M, P(X))$. We establish a certain extension theorem which has a consequence that the mapping $\phi$ is open, and that there is a continuous function $\xi: C(M, P(X)) \rightarrow P(C(M, X))$ such that $\xi(T) \in \phi^{-1}(T)$. (The openness of $\phi$ has been obtained independently by Eifler [7].)

In $\S 3$, we establish a representation theorem when $M$ is a separable metric space, and $X$ is the unit sphere in $R^{2}$.

In $\S 4$, we will see that the conditions on $M$ or $X$ in (a) and (b) can be relaxed and so more general results can be obtained.

2. Extension theorem. In this section, our main task is to give an affirmative answer to the following question:

Let $T \in C(M, P(X))$. Suppose that $F$ is a closed subset of $M$, and that $\mu_{F} \in P(C(F, X))$ representing $\left.T\right|_{F}$. Is there a measure $\mu \in P(C(M, X))$ representing $T$ and $\mu\left\{f \in C(M, X):\left.f\right|_{F} \in L\right\}=\mu_{F}(L)$ for all Borel sets $L \subseteq C(F, X)$ ?

Before giving the basic lemma, we review briefly some notations we need, and give one more piece of notation. Let $M$ be a compact metric space, and let $Y$ and $X$ be Polish spaces. Let $F$ be a closed subset of $M$. Let $i_{F}: F \rightarrow M$ be the natural inclusion map, and let $\pi: Y \rightarrow X$ be a continuous function. Then $\pi$ induces a continuous mapping, also denoted by $\pi$, from $P(Y)$ to $P(X)$ and defined by $\pi \mu(E)=\mu\left(\pi^{-1}(E)\right)$, and another continuous mapping, from $C(M, Y)$ to $C(M, X)$, by $\pi \phi(t)=\pi(\phi(t)), t \in M, \phi \in C(M, Y)$. We denote this mapping by $\pi$ also. The mapping $i_{F}: F \rightarrow M$ induces a continuous mapping, also denote by $i_{F}$, from $C(M, Y) \rightarrow C(F, Y)$, by $i_{F} f(t)=$ $f\left(i_{F}(t)\right)$ for all $t \in F, f \in C(M, Y)$. If we regard $\pi: C(M, Y) \rightarrow C(M, X)$, $i_{F}: C(M, X) \rightarrow C(F, X)$, then $i_{F} \pi: C(M, Y) \rightarrow C(F, X)$. If we regard $i_{F}: C(M, Y) \rightarrow C(F, Y), \pi: C(F, Y) \rightarrow C(F, X)$, then $\pi i_{F}: C(M, Y) \rightarrow$ $C(F, X)$. It is easy to check that $i_{F} \pi=\pi i_{F}$.

LEMMA 2.1. Let $M$ be a totally disconnected compact metric space, let $F$ be a closed subspace of $M$, and let $i_{F}: F \rightarrow M$ be the natural inclusion map. Let $Y$ and $X$ be countable discrete spaces and let $\pi$ be a continuous mapping from $Y$ onto $X$. Let $T$ be a continuous function from $M$ into $P(Y)$, and let $\nu \in P(C(M, X)$ ), $\mu_{F} \in P(C(F, Y))$ be such that

(1) $t \mu_{F}=T(t)$ for all $t \in F$ 
(2) $t \nu=\pi T(t)$ for all $t \in M$

(3) $\pi \mu_{F}=i_{F} \nu$.

Then there is a measure $\mu \in P(C(M, Y))$ such that

(1) $t \mu=T(t)$ for all $t \in M$

(2) $\pi \mu=\nu$

(3) $i_{F} \mu=\mu_{F}$.

Proof. Let $A=\{\theta \mid \theta$ is a measure on $C(M, Y), \pi \theta \leqq \nu, t \theta \leqq T(t)$, $\left.i_{F} \theta \leqq \mu_{F}\right\}$. We will show that $A$ contains a non-zero element. Indeed, $C(M, X)$ is a countable discrete space [6, p. 265] and so there is an element $f \in C(M, X)$ such that $\nu(\{f\})=\varepsilon>0$. Denote the restriction of $f$ on $F$ by $f_{F}$. Then $i_{F} \nu\left(\left\{f_{F}\right\}\right) \geqq \varepsilon$. Since $\pi \mu_{F}=i_{F} \nu$, there is a continuous function $g: F \rightarrow Y$ such that $\pi g=f_{F}$ and $\mu_{F}(\{g\})=\delta>0$. Suppose that $g$ takes on values $x_{1}, \cdots, x_{n}$ on sets $M_{1}, \cdots, M_{n}$ respectively. Since $T(t)=t \mu_{F}$ for all $t \in F$, we have $T(t)\left(\left\{x_{i}\right\}\right) \geqq \delta$ for each $t \in M_{i}$. For each $x_{i}$, the function $t \rightarrow T(t)\left(\left\{x_{i}\right\}\right)$ is continuous. Since $M$ is totally disconnected and compact metric, we may pick pair-wise disjoint both open and closed subsets $U_{1}, \cdots, U_{n}$ of $M$ such that $M_{i} \leqq U_{i}, 1 \leqq i \leqq n, T(t)\left(\left\{x_{i}\right\}\right) \geqq \delta_{0}=(1 / 2) \delta$, for each $t \in U_{i}$, and $f\left(U_{i}\right)=f\left(M_{i}\right)=\pi g\left(M_{i}\right)=\pi\left(x_{i}\right)$. Let $L=\bigcup_{i=1}^{n} U_{i}$, then $L$ is a both open and closed subset of $M$. Define a continuous function $h$ of $L$ into $Y$ by $h(t)=x_{i}$ if $t \in U_{i}, 1 \leqq i \leqq n$. Now we consider the restriction of $f$ on $L^{c}=M \backslash L$, denoted by $f_{L^{c}}$. We also denote the measure $i_{L^{c}} \nu$ by $\nu_{L^{c}}$. Then $\pi T(t)=t \nu_{L^{c}}$ for all $t \in L^{c}$, and $\nu_{L^{c}}\left(f_{L^{c}}\right) \geqq \varepsilon$. By the lemma of [2], there is a continuous function $k: L^{c} \rightarrow Y$ such that $\pi k=f_{L^{c}}$ and a number $\delta^{\prime}>0$ such that if $\theta$ is a point-mass measure at $k$ with mass $\delta^{\prime}$, then $t \theta \leqq T(t)$ for each $t \in L^{\circ}$. Define $\hat{g}: M \rightarrow Y$ by $\hat{g}(t)=h(t)$ if $t \in L$ and $\hat{g}(t)=k(t)$ if $t \in L^{c}$. Then $\hat{g}$ is continuous, and $\pi \hat{g}=f$. Let $\delta_{0}^{\prime}=\operatorname{Min}\left\{\delta_{0}, \delta^{\prime}, \varepsilon\right\}$, and let $\theta_{0}$ be the measure putting mass $\delta_{0}^{\prime}$ at the point $\hat{g} \in C(M, Y)$, then $\theta_{0}$ is nonzero, and it is easy to check that $\theta_{0} \in A$. Now return to the proof of the lemma. The set $A$ is inductively ordered: indeed, if $K$ is a totally ordered subset of $A$, we take $\mu_{1} \leqq \mu_{2} \leqq \cdots$ from $K$ such that $\lim _{n \rightarrow \infty} \mu_{n}(C(M, Y))=\operatorname{Sup}_{\mu_{\in} K} \mu(C(M, Y))$, then $\alpha=\lim _{n} \mu_{n}$ is an element of $A$ and $\alpha \geqq \rho$ for every $\rho \in K$. Let $\theta$ be a maximal element of $A$. If $\theta$ has mass 1 , then $t \theta=T(t), \pi \theta=\nu$ and $i_{F} \theta=\mu_{F}$. If $\theta$ has mass $\eta<1$, then we may apply the first part of the proof to the mapping:

$$
T^{\prime}(t)=\frac{T(t)-t \theta}{1-\eta} \in C(M, P(Y)) \text { and }
$$

measures:

$$
\mu_{F}^{\prime}=\frac{\mu_{F}-i_{F} \theta}{1-\eta} \in P(C(F, Y))
$$




$$
\nu^{\prime}=\frac{\nu-\pi \theta}{1-\eta} \in P(C(M, X)) .
$$

This will yield a strictly positive measure $\theta^{\prime}$ with $t \theta^{\prime} \leqq T^{\prime}(t), \pi \theta^{\prime} \leqq \nu^{\prime}$ and $i_{F} \theta^{\prime} \leqq \mu_{F}^{\prime}$, and then $\theta+(1-\eta) \theta^{\prime}$ will be an element of $A$ strictly exceeding $\theta$. This completes the proof.

LEMMA 2.2. Let $M$ be a totally disconnected compact metric space, and let $F$ be a closed subset of $M$. Let $i_{F}: F \rightarrow M$ be the natural inclusion map. Let $G$ be a totally disconnected complete separable metric space. Let $T \in C(M, P(G))$ and $\mu_{F} \in P(C(F, G))$ be such that $T(t)=t \ell_{F}$ for all $t \in F$. Then there is a measure $\mu \in P\left(C(M, G)\right.$ ) such that $T(t)=$ t $\mu$ for all $t \in M$ and $i_{F} \mu=\mu_{F}$.

Proof. It is easy to check that $G$ can be identified as a closed subspace of a countable product $X_{i=1}^{\infty} F_{i}$ of discrete spaces, with each $F_{i}$ being countable, and $F_{1}$ consisting of a single point. For each $n>0$, let $P_{n}: G \rightarrow F_{1} \times \cdots \times F_{n}$ be defined by $P_{n}\left(\left(g_{1}, \cdots, g_{n}, \cdots\right)\right)=\left(g_{1}, \cdots, g_{n}\right)$. Let $X_{n}=P_{n}(G)$. Then $X_{n}$ is a subspace of $F_{1} \times \cdots \times F_{n}$, and so is a countable discrete space. Let $\pi_{n}: X_{n} \rightarrow X_{n-1}$ be the mapping that sends $\left(g_{1}, \cdots, g_{n-1}, g_{n}\right)$ to $\left(g_{1}, \cdots, g_{n-1}\right)$. Then $\pi_{n} P_{n}=P_{n-1}$. Let $T^{(n)}(t)=P_{n} T(t)$. Then $T^{(n)}$ is a continuous function from $M$ to $P\left(X_{n}\right)$, and $\pi_{n} T^{(n)}=T^{(n-1)}$. Let $\mu_{F}^{(n)}=P_{n} \mu_{F} \in P\left(C\left(F, X_{n}\right)\right)$, and let $T_{F}^{(n)}(t)=t \mu_{F}^{(n)}$. Then $\pi_{n} \mu_{F}^{(n)}=\mu_{F}^{(n-1)}$, and $T^{(n)}(t)=T_{F}^{(n)}(t)$ if $t \in F$. When $n=1$, we have of course the trivial measure $\mu^{(1)}$ putting mass 1 on the one point of $C\left(M, X_{1}\right)$ so that (a) $t \mu^{(1)}=T^{(1)}(t)$ and (b) $i_{F} \mu^{(1)}=\mu_{F}^{(1)}$. Consequently, by repeatedly applying Lemma 2.1 , we obtain a sequence of measures $\mu^{(n)} \in P\left(C\left(M, X_{n}\right)\right)$ such that (a) $t \mu^{(n)}=T^{(n)}(t)$, (b) $i_{F} \mu^{(n)}=$ $\mu_{F}^{(n)}$ and (c) $\pi_{n} \mu^{(n)}=\mu^{(n-1)}$ for all $n$. By Kolomogorov's consistency theorem [4, p. 120], there is a measure $\mu \in P(C(M, G))$ such that $P_{n} \mu=\mu^{(n)}$ for all $n$. Since for all $n, P_{n} t \mu=t \mu^{(n)}=P_{n} T(t)$, and $P_{n} i_{F} \mu=i_{F} \mu^{(n)}=P_{n} \mu_{F}$, so that $t \mu=T(t)$ for all $t \in M$ and $i_{F} \mu=\mu_{F}$. This completes the proof.

THEOREM 2.3. Let $M$ be a totally disconnected compact metric space and let $G$ be a totally disconnected complete separable metric space. Suppose that $\left\{T_{n}\right\}$ is a sequence converging to $T$ in $C(M, P(G))$ and that $\mu$ is a measure in $P(C(M, G)$ ) such that t $\mu=T(t)$ for all t. Then there is a sequence $\left\{\mu_{n}\right\}$ in $P(C(M, G))$ such that $\mu_{n} \rightarrow \mu$ and $t \mu_{n}=T_{n}(t)$ for all $t$. This is equivalent to saying that the natural map $\phi: P((M, G)) \rightarrow C(M, P(G))$ as described in the introduction is open.

Proof. Let $D=\left\{T_{n}: n=1,2, \cdots\right\} \cup\{T\}$ with the subspace 
topology. Then the product space $D \times M$ is totally disconnected compact metric, and the function $\psi: D \times M \rightarrow P(G)$ defined as $\psi(S, t)=S(t)$ is continuous. Consider the closed subspace $F=$ $\{T\} \times M$ of $D \times M$. The measure $\mu$, regarded as a measure on $C(\{T\} \times M, G)$, satisfies $\psi(s)=s \mu$ for every $s \in F$. Therefore, by Lemma 2.2, there is $\tilde{\mu} \in P(C(D \times M, G))$ such that $s \tilde{\mu}=\psi(s)$ for all $s \in D \times M$, and $i_{F} \tilde{\mu}=\mu$. For each $S \in D$, define a measure $S \tilde{\mu} \in$ $P(C(M, G))$ by

$$
S \tilde{\mu}(K)=\tilde{\mu}\{f \in C(D \times M, G): f(S, \cdot) \in K\} .
$$

Then if we set $\mu_{n}=T_{n} \tilde{\mu}$, we obtain $t \mu_{n}=T_{n}(t)$ for all $t \in M$, and $\mu_{n} \rightarrow \mu$ in $P(C(M, G))$. This completes the proof.

Recall that the natural map $\phi: P(C(M, G)) \rightarrow C(M, P(G))$ in the introduction is defined as $\phi(\mu)(t)=t \mu$, for $\mu \in P(C(M, G)), t \in M$. By Theorem 2.3, $\phi$ is an open mapping. Moreover, we have the following:

THEOREM 2.4. There is a continuous function $\xi: C(M, P(G)) \rightarrow$ $P(C(M, G))$ such that $\xi(T) \in \phi^{-1}(T)$ for all $T$.

Proof. Let $\mathscr{C l}(C(M, G))$ be the space of all finite signed measures on $C(M, G)$ with the weak topology as functionals on $C(C(M, G))$. Then, $P(C(M, G))$ is a closed subspace of $\mathscr{C}(C(M, G))$. It is clear that the closed convex hull of every compact subset of $P(C(M, G))$ is compact. Let $2^{P(C(M, G))}$ be the collection of all non-empty subsets of $P(C(M, G))$. Define a function $\tilde{\phi}: C(M, P(G)) \rightarrow 2^{P(C(M, G))}$ by

$$
\tilde{\phi}(T)=\phi^{-1}(T) \text {. }
$$

Since $\phi$ is an open mapping, it follows that $\tilde{\phi}$ is lower semi-continuous [8]. Therefore, there is a continuous function $\xi: C(M, P(G)) \rightarrow$ $P(C(M, G))$ such that $\xi(T) \in \tilde{\phi}(T)=\phi^{-1}(T)$ [8]. This completes the proof.

In the following, we will establish two lemmas from which we will obtain the same results of Lemma 2.2, Theorem 2.3, and Theorem 2.4 in replacing the space $G$ by an arbitrary Polish space $X$.

Lemma 2.5. Let $X$ be a Polish space. Then there is a totally disconnected complete separable metric space $G$, a continuous function $\phi: G \rightarrow X$ and a continuous function $\tilde{\phi}: P(X) \rightarrow P(G)$ such that $\phi \tilde{\phi}(\mu)=\mu$ for all $\mu \in P(X)$. Moreover, $\tilde{\phi}$ is an affine map: 


$$
\tilde{\phi}\left(\frac{1}{2} \mu+\frac{1}{2} \nu\right)=\frac{1}{2} \tilde{\phi}(\mu)+\frac{1}{2} \tilde{\phi}(\nu) \text {. }
$$

Proof. Let $F_{n}$ be a countable partition of unity of $X$, subordinated by a cover of diameter $\leqq 1 / n$. Give $F_{n}$ the discrete topology. Let

$$
X_{n}=\left\{\left(g_{1}, \cdots, g_{n}\right) \mid g_{i} \in F_{i}, g_{1} \cdots g_{n} \neq 0\right\}
$$

and

$$
G=\left\{\left(g_{1}, \cdots, g_{n}, \cdots\right) \mid\left(g_{1}, \cdots, g_{n}\right) \in X_{n} \text { for all } n\right\} .
$$

Give $G$ the subspace topology of the product space $X_{i=1}^{\infty} F_{i}$. Then $G$ is a totally disconnected, complete separable metric space. Define $\phi: G \rightarrow X$ by $\phi\left(g_{1}, \cdots, g_{n}, \cdots\right)$ to be the unique point $x \in \operatorname{Supp} g_{n}$ for all $n$. Then $\phi$ is continuous. Let $P_{n}: G \rightarrow X_{n}$ be the canonical projection. Define $\tilde{\phi}: P(X) \rightarrow P(G)$ by setting $\tilde{\phi}(\mu)$ to be the unique measure $\tilde{\mu} \in P(G)$ such that $P_{n} \tilde{\mu}\left\{\left(g_{1}, \cdots, g_{n}\right)\right\}=\int g_{1} \cdots g_{n} d \mu$, for all $\left(g_{1}, \cdots, g_{n}\right)$ in $X_{n}$ and for all $n$ (via the Kolomogorov consistency theorem [4, p. 120]). It is clear that $\tilde{\phi}$ is continuous and $\phi \tilde{\phi}(\mu)=\mu$ for all $\mu \in P(X)$. It is also easy to check that

$$
\tilde{\phi}\left(\frac{1}{2} \mu+\frac{1}{2} \nu\right)=\frac{1}{2} \tilde{\phi}(\mu)+\frac{1}{2} \tilde{\phi}(\nu) \text {. }
$$

The proof is complete.

Lemma 2.6. Let $X, G, \phi, \tilde{\phi}$ be as in Lemma 2.5. Let $T \in C(M, P(X)), \mu \in P(C(M, X))$ be such that $T(t)=$ te for all $t$. Then there is a measure $\hat{\mu} \in P(C(M, G))$ such that $\tilde{\phi} T(t)=t \hat{\mu}$ for all $t$, and $\phi \hat{\mu}=\mu$.

Proof. Since $C(M, X)$ is a polish space, there is a compact subset $L$ of $C(M, X)$ such that $\mu(L) \geqq 1-\varepsilon$. We may assume that Supp $\mu$ is a compact subset $L$ of $C(M, X)$. Each $f \in L$ is regarded as a continuous function from $M$ to $P(X)$. Let $\tilde{f}=\tilde{\phi} f$, then $\tilde{f} \in C(M, P(G))$. Let $\xi: C(M, P(G)) \rightarrow P(C(M, G))$ be a continuous function as in Theorem 2.4. Denote $\xi(\widetilde{f})$ by $\hat{\mu}_{f}$, then $t \hat{\mu}_{f}=\widetilde{f}(t)$ for all $t$. Let $\mu_{f}=\phi \hat{\mu}_{f}$, then $t \mu_{f}=f(t)$ for all $t$. Therefore, $\mu_{f}=\varepsilon_{f}$, the unit point-mass measure putting mass 1 on the point $f \in C(M, X)$. Since $L$ is compact and the function $f \rightarrow \hat{\mu}_{f}$ is continuous, the set $\left\{\hat{\mu}_{f} \mid f \in L\right\}$ is compact. Hence for $\varepsilon>0$, there is a compact set $\widetilde{L} \cong C(M, G)$ such that $\hat{\mu}_{f}(\widetilde{L})>1-\varepsilon$ for all $f \in L$ [9, p. 47]. Now $\mu \in P(L)$, there is a sequence $\mu_{n} \rightarrow \mu$ in $P(L)$ with $\mu_{n}=\sum_{i=1}^{k} \lambda_{i} \varepsilon_{f_{i}}$, for some $\lambda_{i}>0, \sum \lambda_{i}=1$ and $f_{i} \in L$. Let $\hat{\mu}_{n}=\sum_{i=1}^{k} \lambda_{i} \hat{\mu}_{f_{i}}$, then 
$\phi \hat{\mu}_{n}=\mu_{n}$ and

$$
\begin{aligned}
t \widehat{\mu}_{n} & =\sum_{i=1}^{k} \lambda_{i} \widetilde{f}_{i}(t)=\sum_{i=1}^{k} \lambda_{i} \tilde{\phi}\left(f_{i}(t)\right) \\
& =\tilde{\phi}\left(\sum_{i=1}^{k} \lambda_{i} \varepsilon_{f_{i}(t)}\right)=\tilde{\phi}\left(T_{n}(t)\right)
\end{aligned}
$$

for all $t$, where $T_{n}(t)=t \mu_{n}$. Since $\mu_{n} \rightarrow \mu$, so that $T_{n}(t) \rightarrow T(t)$ in $P(X)$. Also, $\hat{\mu}_{n}(\widetilde{L})>1-\varepsilon$ for all $n$. Hence $\left\{\hat{\mu}_{n}: n=1,2,3, \cdots\right\}$ is relative compact. Therefore some subsequence $\hat{\mu}_{n_{k}} \rightarrow \hat{\mu}$ in $P(C(M, G))$. Since $\tilde{\phi}\left(T_{n_{k}}(t)\right)=t \hat{\mu}_{n_{k}}$ and $\phi\left(\hat{\mu}_{n_{k}}\right)=\mu_{n_{k}}$, letting $k \rightarrow \infty$, we obtain $\tilde{\phi}(T(t))=t \hat{\mu}$ for all $t$, and $\phi \hat{\mu}=\mu$. This completes the proof.

THEOREM 2.7 (Extension theorem). Let $M$ be a totally disconnected compact metric space, $F$ be a closed subset of $M$, and $i_{F}: F \rightarrow M$ be the natural inclusion. Let $X$ be a Polish space. Let $T$ be a continuous function from $M$ into $P(X)$, and $\mu_{F}$ be a measure in $P\left(C(F, X)\right.$ ) such that $t \mu_{F}=T(t)$ for all $t \in F$. Then there is a measure $\mu \in P(C(M, X))$ such that $T(t)=t \mu$ for all $t \in M$ and $i_{F} \mu=\mu_{F}$.

Proof. By Lemmas 2.5 and 2.6, there is a totally disconnected complete separable metric space $G$ and there are continuous functions $\phi: G \rightarrow X, \tilde{\phi}: P(X) \rightarrow P(G)$ and a measure $\hat{\mu}_{F} \in P(C(F, G))$ such that $\phi \hat{\mu}_{F}=\mu_{F}$ and $t \hat{\mu}_{F}=\tilde{\phi}(T(t))$ for all $t \in F$. By Lemma 2.2, there is a measure $\hat{\mu} \in P(C(M, G))$ such that $t \hat{\mu}=\tilde{\phi}(T(t))$ for all $t \in M$, and $i_{F} \hat{\mu}=\hat{\mu}_{F}$. Let $\mu=\phi \hat{\mu}$. Then $\mu$ is a measure in $P(C(M, X))$ such that $t \mu=\phi(\tilde{\phi} T(t))=T(t)$ for all $t \in M$ and $i_{F} u=\mu_{F}$. This completes the proof.

Therefore, by following the same arguments as in Theorem 2.3 and Theorem 2.4, we obtain:

THEOREM 2.8. Let $M$ be a totally disconnected compact metric space, and let $X$ be a Polish space. Then the natural map $\phi: P(C(M, X)) \rightarrow C(M, P(X))$, defined as $\phi(\mu)(t)=t \mu$, is open, and there is a continuous function $\xi: C(M, P(X)) \rightarrow P(C(M, X))$ such that $\xi(T) \in \phi^{-1}(T)$ for all $T$.

As an application of Theorem 2.7, we obtain:

CoROLlary 2.9. Let $M$ and $X$ be as in Theorem 2.8, and let $T: M \times M \rightarrow P(X \times X)$ be continuous. Let $\Delta_{M}=\{(t, t): t \in M\}$. For any subset $A \subseteq X$, let $\Delta_{A}=\{(a, a): a \in A\}$. Suppose $\mu_{\Delta}$ is a measure in $P\left(C(M, X)\right.$ ) such that $t \mu_{A}(A)=T(t, t)\left(\Delta_{A}\right)$ for all $t \in M$. Regard $\mu_{A}$ 
as a measure on $C\left(\Delta_{M}, \Delta_{X}\right)$. Then there is a measure $\mu \in P(C(M \times$ $M, X \times X)$ ) representing $T$ such that $i_{\Delta} \mu=\mu_{\Delta}$, where $i_{\Delta}: \Delta_{M} \rightarrow M \times M$ is the natural inclusion.

We remark that the function $\xi$ in Theorem 2.8 is a continuous selection function which selects for each $T$ in $C(M, P(X))$ a measure $\mu_{T}(=\xi(T))$ in $P(C(M, X))$ representing $T$.

\section{Representation when $X=S^{1}$}

THEOREM 3.1. Let $M$ be a separable metric space, and let $S^{1}$ be the unit sphere in $R^{2}$. Let $0<k \leqq 2 \pi$, and let $T: M \rightarrow P\left(S^{1}\right)$ be a continuous function such that for each $t \in M$, Supp $T(t)$ is connected and of arc length $\leqq 2 \pi-k$. Then there is a measure $\mu \in P\left(C\left(M, S^{1}\right)\right)$ such that $T(t)=$ te for all $t$.

Proof. Let $P_{k}\left(S^{1}\right)=\left\{\mu \in P\left(S^{1}\right)\right.$ : $\operatorname{Supp}(\mu)$ is connected and has arc length at most $2 \pi-k\}$. Given $r<t$, set $(r, t)=\left\{e^{i s}: r<s<t\right\}$ and set $[r, t]=\left\{e^{i s}: r \leqq s \leqq t\right\}$. Following an idea in [3, Lemma 2.1], we construct a mapping $Z: P_{k}\left(S^{1}\right) \times(0,1) \rightarrow S^{1}$ such that

$$
P\{\omega: Z(\mu, \omega) \in(a, b)\}=\mu(a, b)
$$

for each $a<b$ where $P$ is Lebesgue measure. Namely, given $\mu \in P_{k}\left(S^{1}\right)$, choose $\alpha_{\mu}$ and $b_{\mu}$ such that $\operatorname{Supp}(\mu)=\left[\alpha_{\mu}, b_{\mu}\right]$ and set $Z(\mu, \omega)=e^{i s}$ where $s=\inf \left\{t \geqq a_{\mu}: \mu\left[\alpha_{\mu}, t\right] \geqq \omega\right\}$. We see that $Z(\mu, \omega)$ is well-defined since $\operatorname{Supp}(\mu) \neq S^{1}$. Suppose $\mu_{n} \rightarrow \mu$ where $\mu_{n} \in P_{k}\left(S^{1}\right)$. Set $I_{n}=S^{1} \backslash \operatorname{Supp}\left(\mu_{n}\right)$ and set $S^{1} \backslash \operatorname{Supp}(\mu)=(a, b)$. Set $J=$ $(a+k / 3, b-k / 3)$. By considering the geometry of $S^{1}$, we see that $J \subseteq \operatorname{Supp}\left(\mu_{n}\right)$ for each $n$ is impossible. Hence, $J \cap I_{n} \neq \varnothing$ for $n$ large. One may check that if $\mu_{n} \rightarrow \mu$, that $Z\left(\mu_{n}, \omega\right) \rightarrow Z(\mu, \omega)$. One checks directly that $(*)$ holds.

Let $\Omega$ be the open interval $(0,1)$. For each $\omega \in \Omega, t \in M$, define $X_{t}(\omega)$ by $X_{t}(\omega)=Z(T(t), \omega)$. Thus, for a fixed $\omega \in \Omega$, the function $t \rightarrow X_{t}(\omega)$ is a continuous function from $M$ into $S^{1}$. Therefore, we may define a mapping $X: \Omega \rightarrow C\left(M, S^{1}\right)$ by $X(\omega)(t)=X_{t}(\omega)$. One checks easily that $X$ is Borel measurable and $P\left\{\omega: X_{t}(\omega) \in(a, b)\right\}=$ $T(t)(a, b)$ for any arc $(a, b)$ in $S^{1}$. Define a measure $\mu \in P\left(C\left(M, S^{1}\right)\right)$ by $\mu(A)=P\left(X^{-1}(A)\right)$. Then $t \mu=T(t)$ for all $t$. This completes the proof.

\section{EXAMPLE 3.2.}

(a) This example shows that the requirement of an upper bound $2 \pi-k<2 \pi$ for the lengths of all Supp $T(t)$ cannot be entirely eliminated. 
Let $M=D^{1}=\left\{x \in R^{2}:|x| \leqq 1\right\}, X=S^{1}$, and let $T$ be a continuous function from $M$ into $P\left(S^{1}\right)$ such that $T(t)=\varepsilon_{t}$, the unit point mass measure at the point $t$, for each $t \in S^{1}$. Then any measure representing $T$ must attribute all its mass to those continuous functions from $D^{1}$ to $S^{1}$ which are the identity on $S^{1}$. Since there is no such function, no such $T$ has a representing measure. An example of such a $T$ in which each $T(t)$ is supported by a subarc of $S^{1}$ is:

$$
\begin{aligned}
T\left(r e^{i t}\right)= & r^{2} \varepsilon_{t}+\left[\left(1-r^{2}\right)-\left(\frac{2 \pi-t}{2 \pi}\right)\left(r-r^{2}\right)\right] \varepsilon_{0} \\
& +\left(\frac{2 \pi-t}{2 \pi}\right)\left(r-r^{2}\right) l_{[0, t]}, \quad 0 \leqq r \leqq 1, \quad 0 \leqq t \leqq 2 \pi,
\end{aligned}
$$

where $l_{[0, t]}$ is the probability measure uniformly distributed on the arc $[0, t]=\left\{e^{i s}: 0 \leqq s \leqq t\right\}$.

Note that there is no bound on the arc lengths of the supports as required for Theorem 3.1.

(b) The following example shows that for a continuous function $T$ from $S^{1}$ into $P\left(S^{1}\right)$, all supports of $T(t)$ connected is not enough for representation.

Define $T: S^{1} \rightarrow P\left(S^{1}\right)$ as follows:

We regard $S^{1}$ as the quotient space by identifying 0 and $2 \pi$ in the closed interval $[0,2 \pi]$.

Let

$$
t_{n}=\left(\frac{2^{n}-2}{2^{n}}\right) 2 \pi \quad n=1,2,3, \cdots .
$$

Define $T\left(t_{n}\right)=\varepsilon_{t_{n}}$, and

$$
\begin{aligned}
T\left(\frac{t_{n}+t_{n+1}}{2}\right)= & \frac{1}{2^{n}} l_{\left(S^{1} \backslash J_{n}{ }^{\circ}\right)}+\frac{\left(1-\frac{1}{2^{n}}\right)}{2} T\left(t_{n}\right) \\
& +\frac{\left(1-\frac{1}{2^{n}}\right)}{2} T\left(t_{n+1}\right),
\end{aligned}
$$

where $J_{n}^{\circ}=\left\{e^{i t}: t_{n}<t<t_{n+1}\right\}$ and $l_{S^{1} \backslash J_{n}^{\circ}}$ is the probability measure uniformly distributed on $S^{1} \backslash J_{n}^{\circ}$. Define $T$ piece-wise linear on $\left[t_{n},\left(t_{n}+t_{n+1}\right) / 2\right]$ and on $\left[\left(t_{n}+t_{n+1}\right) / 2, t_{n+1}\right]$. Define $T(2 \pi)=T(0)=\varepsilon_{0}$. Then $T$ is continuous and $\operatorname{Sup} T(t)$ is connected for each $t$. Let $A_{n}=\left\{f \in C\left(S^{1}, S^{1}\right) \mid f\left(t_{n}\right)=t_{n}, \quad f\left(t_{n+1}\right)=t_{n+1}, f\left(t_{n}, t_{n+1}\right) \cap\left(t_{n}, t_{n+1}\right)=\varnothing\right\}$. Note that if $\mu$ represents $T$, then $\mu\left(A_{n}\right)=1$. Therefore, $\mu\left(\overline{\lim _{n}} A_{n}\right)=1$. But $\overline{\lim }_{n} A_{n}=\varnothing$. Therefore, $T$ cannot be represented by a measure.

4. Some generalizations, We will present here some results 
for our representation theorem. As you will see, most of them generalize the results of (a) and (b) as described in the introduction. The proofs of these theorems are, by no means, difficult, and they can be found in [5].

THEOREM 4.1. Let $M$ be a compact extremally disconnected space, and let $X$ be a Prohorov space. Let $T: M \rightarrow P_{\tau}(X)$ be a continuous function. Then there is a tight measure $\mu \in P(C(M, X))$ such that $T(t)=t \mu$ for all $t$.

THEOREM 4.2. Let $M$ be a separable metric space, and let $I$ be the unit interval. Let $T: M \rightarrow P(I)$ be continuous such that Supp $T(t)$ is a connected subset of $I$ for all $t$. Then there is a measure $\mu \in P(C(M, X))$ such that $T(t)=t \mu$ for all $t$.

THEOREM 4.3. Let $M$ be a separable metric space, and let $X$ be a Peano space. Let $T: M \rightarrow P(X)$ be continuous such that the set $M_{1}=\{t \in M$ : Supp $T(t) \neq X\}$ is at most countable. Then there is a measure $\mu \in P(C(M, X))$ such that $T(t)=$ t $\mu$ for all $t$.

THEOREM 4.4. Let $M$ be a separable metric space, and let $X$ be a metric space such that there is a sequence of open sets $U_{i}$ with compact closures $K_{i}$ satisfying

(a) $U_{i} \cong K_{i} \subseteq U_{i+1}$ for all $i$,

(b) $K_{i}$, with the relative topology, is a Peano space, and

(c) $X=\bigcup_{i=1}^{\infty} K_{i}$.

(e.g., $X=R^{n}$, for any integer $n>0$.)

Let $T$ be a continuous function from $M$ into $P(X)$ such that Supp $T(t)=X$ for all $t$. Then there is a measure $\mu \in P(C(M, X))$ such that $T(t)=t \mu$ for all $t$.

TheOREM 4.5. Let $M$ be a countable metric space, and let $X$ be a metric space. Let $T: M \rightarrow P_{\tau}(X)$ be continuous. Then there is a measure $\mu \in P(C(M, X))$ such that $T(t)=t \mu$ for all $t$. As a consequence, for any countable closed subset $F$ of $P_{\tau}(X), F$ is compact if and only if $F$ is tight.

One may wonder how far we can go in this representation theorem. Among the difficulties, one notes that the functions in $C(M, X)$ are not enough in general. However, the following question remains unknown, and is worthy to work with:

Let $M$ be a totally disconnected compact metric space, and let $X$ be a Prohorov space. Let $T$ be a continuous function from $M$ 
into $P_{\tau}(X)$. Is there a measure $\mu \in P(C(M, X))$ such that $T(t)=t \mu$ for all $t$ ?

ACKNOWLEDGMENT. This paper is part of the author's $\mathrm{Ph} . \mathrm{D}$. thesis prepared under the supervision of Professor Robert M. Blumenthal. The author wishes to acknowledge his indebtedness to him for many fine suggestions and constructive criticisms for the preparation of this paper.

\section{REFERENCES}

1. P. Billingsley, Convergence of Probability Measures, Wiley, New York, (1968), 240.

2. R. M. Blumenthal and H. H. Corson, On continuous collections of measures, Des Annals de L'Institute Fourier de L'Universite de Grenoble, TOMXX-F2, (1970), 193-199. 3. - On continuous collections of measures, Sixth Berkeley Symposium on Mathematical Statistics and Probability, 2, (1972), 33-40.

4. S. Bochner, Harmonic Analysis and the Theory of Probability, University of California Press, Berkeley, 1955.

5. S. M. Chang, Measures with continuous image law, Ph. D. thesis, University of Washington, 1975.

6. J. Dugundji, Topology, Allyn and Bacon, Boston, 1966.

7. L. Q. Eifler, Open mapping theorems for probability measures on metric spaces, to appear in Pacific J. Math.

8. E. A. Michael, Three mapping theorems, Amer. Math. Soc. Proceedings, 15, (1964), 410-416.

9. K. R. Parthasarathy, Probability Measures on Metric Spaces, Academic Press, New York and London, 1967.

Received January 22, 1976 and in revised form October 13, 1976.

UNIVERSITY OF TORONTO

AND

UNIVERSITY OF WASHINGTON 


\section{PACIFIC JOURNAL OF MATHEMATICS}

EDITORS

RICHARD ARENS (Managing Editor)

University of California

Los Angeles, California 90024

R. A. Beaumont

University of Washington

Seattle, Washington 98105
J. DUGUNDJI

Department of Mathematics

University of Southern Californı

Los Angeles, California 90007

D. Gilbarg and J. Milgram

Stanford University

Stanford, California 94305

\section{ASSOCIATE EDITORS}

E. F. BECKENBACH

B. H. NeumanN

F. WOLF

K. YoSHIDA

\section{SUPPORTING INSTITUTIONS}

UNIVERSITY OF BRITISH COLUMBIA

CALIFORNIA INSTITUTE OF TECHNOLOGY

UNIVERSITY OF CALIFORNIA

MONTANA STATE UNIVERSITY

UNIVERSITY OF NEVADA

NEW MEXICO STATE UNIVERSITY

OREGON STATE UNIVERSITY

UNIVERSITY OF OREGON

OSAKA UNIVERSITY
UNIVERSITY OF SOUTHERN CALIFORNIA

STANFORD UNIVERSITY

UNIVERSITY OF TOKYO

UNIVERSITY OF UTAH

WASHINGTON STATE UNIVERSITY

UNIVERSITY OF WASHINGTON

AMERICAN MATHEMATICAL SOCIETY

NAVAL WEAPONS CENTER 


\section{Pacific Journal of Mathematics}

Vol. 69 , No. 1

May, 1977

V. V. Anh and P. D. Tuan, On starlikeness and convexity of certain analytic

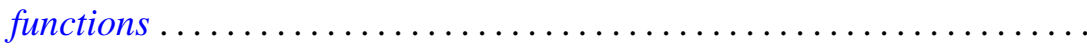

Willard Ellis Baxter and L. A. Casciotti, Rings with involution and the prime

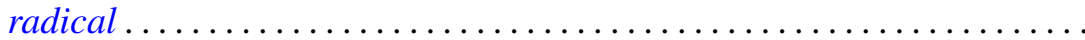

Manuel Phillip Berriozabal, Hon-Fei Lai and Dix Hayes Pettey,

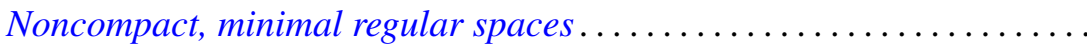

Sun Man Chang, Measures with continuous image law ................

John Benjamin Friedlander, Certain hypotheses concerning

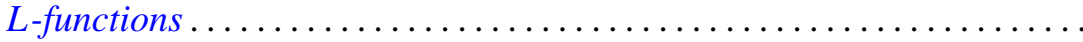

Moshe Goldberg and Ernst Gabor Straus, On characterizations and

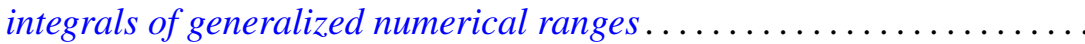

Pierre A. Grillet, On subdirectly irreducible commutative semigroups ...... 55

Robert E. Hartwig and Jiang Luh, On finite regular rings ..............

Roger Hugh Hunter, Fred Richman and Elbert A. Walker, Finite direct sums of cyclic valuated p-groups ........................... 97

Atsushi Inoue, On a class of unbounded operator algebras. III ......... 105

Wells Johnson and Kevin J. Mitchell, Symmetries for sums of the Legendre

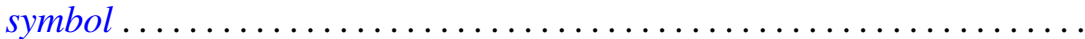

Jimmie Don Lawson, John Robie Liukkonen and Michael William Mislove,

Measure algebras of semilattices with finite breadth

Glenn Richard Luecke, A note on spectral continuity and on spectral properties of essentially $G_{1}$ operators ...............

Takahiko Nakazi, Invariant subspaces of weak-* Dirichlet algebras . .

James William Pendergrass, Calculations of the Schur group ...

Carl Pomerance, On composite $n$ for which $\varphi(n) \mid n-1$. II. . .

Marc Aristide Rieffel and Alfons Van Daele, A bounded operator approach to Tomita-Takesaki theory........................

Daniel Byron Shapiro, Spaces of similarities. IV. $(s, t)$-families ...

Leon M. Simon, Equations of mean curvature type in 2 independent variables.

Joseph Nicholas Simone, Metric components of continuous images of ordered compacta ............................

William Charles Waterhouse, Pairs of symmetric bilinear forms in

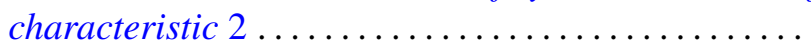

